\title{
SOME NONEXISTENCE RESULTS FOR A SEMIRELATIVISTIC SCHRÖDINGER EQUATION WITH NONGAUGE POWER TYPE NONLINEARITY
}

\author{
TAKAHISA INUI
}

(Communicated by Joachim Krieger)

\begin{abstract}
We consider the following semirelativistic nonlinear Schrödinger equation (SNLS):

$$
\begin{cases}i \partial_{t} u \pm\left(m^{2}-\Delta\right)^{1 / 2} u=\lambda|u|^{p}, & (t, x) \in[0, T) \times \mathbb{R}^{d} \\ u(0, x)=u_{0}(x), & x \in \mathbb{R}^{d}\end{cases}
$$

where $m \geq 0, \lambda \in \mathbb{C} \backslash\{0\}, d \in \mathbb{N}, T>0$, and $\partial_{t}=\partial / \partial t$. Here $\left(m^{2}-\Delta\right)^{1 / 2}:=$ $\mathcal{F}^{-1}\left(m^{2}+|\xi|^{2}\right)^{1 / 2} \mathcal{F}$, where $\mathcal{F}$ denotes the Fourier transform. Fujiwara and Ozawa proved the nonexistence of global weak solutions to SNLS for some initial data in the case of $d=1, m=0$, and $1<p \leq 2$ by a test function method. In this paper, we extend their result to a more general setting: for example, $m \geq 0, d \in \mathbb{N}$, or $p>1$. Moreover, we obtain the upper estimates of weak solutions to SNLS. The key to the proof is to choose an appropriate test function.
\end{abstract}

\section{INTRODUCTION}

We consider the following semirelativistic nonlinear Schrödinger equation:

$$
\begin{cases}i \partial_{t} u \pm\left(m^{2}-\Delta\right)^{1 / 2} u=\lambda|u|^{p}, & (t, x) \in[0, T) \times \mathbb{R}^{d}, \\ u(0, x)=u_{0}(x), & x \in \mathbb{R}^{d},\end{cases}
$$

where $m \geq 0, \lambda \in \mathbb{C} \backslash\{0\}, p>1, d \in \mathbb{N}, T>0$, and $\partial_{t}=\partial / \partial t$. Here $\left(m^{2}-\Delta\right)^{1 / 2}:=$ $\mathcal{F}^{-1}\left(m^{2}+|\xi|^{2}\right)^{1 / 2} \mathcal{F}$, where $\mathcal{F}$ denotes the Fourier transform defined by

$$
(\mathcal{F} f)(\xi)=\hat{f}(\xi):=\frac{1}{(2 \pi)^{d / 2}} \int_{\mathbb{R}^{d}} e^{-i x \cdot \xi} f(x) d x .
$$

In this paper, we are interested in the global existence of solutions to the Cauchy problem (1.1). The following semirelativistic Schrödinger equation appears in many physical contexts:

$$
i \partial_{t} u \pm\left(m^{2}-\Delta\right)^{1 / 2} u=\lambda|u|^{p-1} u,(t, x) \in[0, T) \times \mathbb{R}^{d} .
$$

The local well-posedness and the global solvability for (1.2) are discussed in [1, 3, 8 . However, the global solvability for (1.1) is not well known. It is known that the

Received by the editors March 19, 2015.

2010 Mathematics Subject Classification. Primary 35Q55.

Key words and phrases. Semirelativistic equations, test function, nonexistence of global solution. 
following two equations are quite different from the view point of global solvability:

$$
\begin{aligned}
& \begin{cases}i \partial_{t} u+\Delta u=\lambda|u|^{p-1} u, & (t, x) \in[0, T) \times \mathbb{R}^{d}, \\
u(0, x)=u_{0}(x), & x \in \mathbb{R}^{d},\end{cases} \\
& \begin{cases}i \partial_{t} u+\Delta u=\lambda|u|^{p}, & (t, x) \in[0, T) \times \mathbb{R}^{d}, \\
u(0, x)=u_{0}(x), & x \in \mathbb{R}^{d} .\end{cases}
\end{aligned}
$$

Indeed, if $1<p<1+4 / d, \lambda>0$ and $u_{0} \in L^{2}\left(\mathbb{R}^{d}\right)$, then the corresponding solution to (1.3) is global (see 2 for more information). On the other hand, there exists a blow-up solution for (1.4) even if $u_{0}$ is sufficiently small (see 5, 7, and see 4 for some other nonexistence results). We expect that the global solvability of (1.1) and (1.2) is different. Recently, Fujiwara and Ozawa studied the global solvability of (1.1) in their paper [3. They proved the nonexistence of global weak solutions to (1.1) for some initial data in the case of $d=1,1<p \leq 2$, and $m=0$ by a test function method. To avoid the difficulty which comes from the nonlocal operator $(-\Delta)^{1 / 2}$, they reduced (1.1) with $m=0$ to the following nonlinear wave equation with a time derivative nonlinearity:

$$
\partial_{t}^{2} v-\Delta v=-|\lambda|^{2} \partial_{t}\left(|u|^{p}\right), \quad(t, x) \in[0, T) \times \mathbb{R}^{d},
$$

where $v=\operatorname{Im}(\bar{\lambda} u)$. The wave equation with the nonlinearity $|v|^{p}$ is well studied (see [9, 11, 13] and references therein). However, (1.5) has the difficulty which comes from the time derivative nonlinearity $\partial_{t}\left(|u|^{p}\right)$. (1.5) is more difficult than the damped wave equation, which has time derivative linear term $\partial_{t} u$. The damped wave equation is studied in 6, 10, 12. Because of the difficulty, Fujiwara and Ozawa cannot treat the general case, for instance, $d>1, m>0$ or $p>2$. In the present paper, we extend their result in 3 to the more general case by choosing an appropriate test function and initial data. Moreover, we obtain the upper estimates of the lifespan of weak solutions to (1.1). To state our main results, we introduce some definitions and notation.

$H^{s}=H^{s}\left(\mathbb{R}^{d}\right)$ is the usual inhomogeneous Sobolev space for $s \geq 0$. (.|.) denotes the $L^{2}$ inner product. $a \wedge b:=\min \{a, b\}$ for $a, b \in \mathbb{R}$. We define the function space $X_{T}$ for $T>0$ as follows:

$X_{T}:=\left\{\psi \in C\left([0, \infty) ; H^{1}\right) \cap C^{1}\left([0, \infty) ; L^{2}\right): \operatorname{supp} \psi \subset[0, T) \times \mathbb{R}^{d}, \psi\right.$ is $\mathbb{R}$-valued $\}$.

We define the weak solution to (1.1).

Definition 1.1. Let $s \geq 0, u_{0} \in H^{s}\left(\mathbb{R}^{d}\right)$, and $T>0$. We say that $u$ is a weak solution to (1.1) on $[0, T)$, if $|u|^{p}$ belongs to $L_{l o c}^{1}\left([0, T) ; L^{2}\right)$ and the identity

$$
\int_{0}^{T}\left(u(t) \mid i \partial_{t} \varphi \pm\left(m^{2}-\Delta\right)^{1 / 2} \varphi\right) d t=i(u(0) \mid \varphi(0))+\lambda \int_{0}^{T}\left(|u(t)|^{p} \mid \varphi(t)\right) d t
$$

holds for any $\varphi \in X_{T}$ where the plus-minus sign corresponds to the sign of (1.1).

Based on the idea of Fujiwara and Ozawa [3], we reduce (1.1) to the Klein-Gordon equation with time derivative nonlinearity:

$$
\partial_{t}^{2} v-\Delta v+m^{2} v=-|\lambda|^{2} \partial_{t}\left(|u|^{p}\right), \quad(t, x) \in[0, T) \times \mathbb{R}^{d},
$$


where $v=\operatorname{Im}(\bar{\lambda} u)$. Formally speaking, this is derived by combining the first equation below with $\partial_{t}$ and the latter equation with $\left(m^{2}-\Delta\right)^{1 / 2}$ :

$$
\begin{aligned}
& \partial_{t} \operatorname{Im}(\bar{\lambda} u) \mp\left(m^{2}-\Delta\right)^{-1 / 2} \operatorname{Re}(\bar{\lambda} u)=-|\lambda|^{2}|u|^{p}, \\
& \partial_{t} \operatorname{Re}(\bar{\lambda} u) \pm\left(m^{2}-\Delta\right)^{-1 / 2} \operatorname{Im}(\bar{\lambda} u)=0,
\end{aligned}
$$

which are obtained by taking the real part and the imaginary part after multiplying $\bar{\lambda}$ by (1.1), respectively. We define the weak solution to (1.7).

Definition 1.2. Let $s \geq 0, u_{0} \in H^{s}\left(\mathbb{R}^{d}\right)$, and $T>0$. We say that $u$ is a weak solution to (1.7) on $[0, T)$, if $v=\operatorname{Im}(\bar{\lambda} u),|u|^{p}$ belongs to $L_{l o c}^{1}\left([0, T) \times \mathbb{R}^{d}\right)$, and the identity

$$
\begin{aligned}
& \int_{0}^{T}\left(v(t) \mid \partial_{t}^{2} \varphi-\Delta \varphi+m^{2} \varphi\right) d t \\
= & \pm\left(\operatorname{Re}\left(\bar{\lambda} u_{0}\right) \mid\left(m^{2}-\Delta\right)^{1 / 2} \varphi(0)\right)-\left(v(0) \mid \partial_{t} \varphi(0)\right)+|\lambda|^{2} \int_{0}^{T}\left(|u(t)|^{p} \mid \partial_{t} \varphi(t)\right) d t
\end{aligned}
$$

holds for any $\varphi \in C_{0}^{2}\left([0, T) \times \mathbb{R}^{d}\right)$ where the plus-minus sign corresponds to the sign of (1.1).

Indeed, the above formal argument is justified in the weak sense as follows.

Lemma 1.1. Let $s>0, u_{0} \in H^{s}\left(\mathbb{R}^{d}\right)$, and $T>0$. If $u$ is a weak solution to (1.1) on $[0, T)$, then $u$ is also a weak solution to (1.7) on $[0, T)$.

Lemma 1.1 was essentially proved in 3]. However, we give a complete proof in Appendix $\mathrm{A}$ for the reader's convenience.

Now, we obtain the following main theorems.

Theorem 1.2 (Large data blow-up in the subcritical case). Let $s \geq 0, u_{0} \in$ $H^{s}\left(\mathbb{R}^{d}\right)$, and $T>0$. Let $u$ be a weak solution to (1.1) on $[0, T)$. We assume that $1<p \leq 1+2 /(d-2 s)$ and the initial value $u_{0}(x)=\mu f(x)$ where $\mu>0$ and $f$ satisfies

$$
\operatorname{Re}(\bar{\lambda} f)=0, \quad-\operatorname{Im}(\bar{\lambda} f) \geq \begin{cases}|x|^{-k}, & |x| \leq 1, \\ 0, & |x|>1,\end{cases}
$$

where $k<d / 2-s(\leq 1 /(p-1))$. Then there exists $\mu_{0}$ such that if $\mu>\mu_{0}$, then the weak solution is not global, i.e. $T<\infty$. Moreover, for any $\mu \in\left[\mu_{0}, \infty\right)$, there exists a positive constant $C>0$ such that

$$
T \leq C \mu^{-1 / \kappa},
$$

where $\kappa:=p /(p-1)-k-1>0$.

Theorem 1.3 (Nonexistence of weak solutions in the supercritical case). Let $s \geq 0$, $u_{0} \in H^{s}\left(\mathbb{R}^{d}\right)$, and $T>0$. Let $u$ be a weak solution to (1.1) on $[0, T)$. We assume that $p>1+2 /(d-2 s)$ and the initial value $u_{0}(x)=\mu f(x)$ where $\mu \geq 0$ and $f$ satisfies

$$
\operatorname{Re}(\bar{\lambda} f)=0, \quad-\operatorname{Im}(\bar{\lambda} f) \geq \begin{cases}|x|^{-k}, & |x| \leq 1, \\ 0, & |x|>1,\end{cases}
$$

where $1 /(p-1)<k<d / 2-s$. Then $\mu=0$, i.e. $u_{0} \equiv 0$. 
Theorem 1.4 (Small data blow-up in the mass subcritical and massless case). Let $s \geq 0, u_{0} \in H^{s}\left(\mathbb{R}^{d}\right)$, and $T>0$. Let $u$ be a weak solution to (1.1) on $[0, T)$. We assume that $m=0,1<p<1+2 / d$, and the initial value $u_{0}(x)=\mu f(x)$ where $\mu>0$ and $f$ satisfies

$$
\operatorname{Re}(\bar{\lambda} f)=0, \quad-\operatorname{Im}(\bar{\lambda} f) \geq \begin{cases}0, & |x| \leq 1 \\ |x|^{-k}, & |x|>1,\end{cases}
$$

where $1 /(p-1)<k<d / 2$. Then the weak solution is not global, i.e. $T<\infty$. Moreover, there exist $\varepsilon_{0}>0$ and a positive constant $C>0$ such that

$$
T \leq \begin{cases}C \mu^{-1 / \kappa}, & \mu \in\left(0, \varepsilon_{0}\right), \\ 2, & \mu \in\left[\varepsilon_{0}, \infty\right),\end{cases}
$$

where $\kappa:=p /(p-1)-k-1>0$.

The proofs are based on a test function method used in [3] 6 . The key to our proof is to choose an appropriate test function.

We state the construction of the present paper: In Section 2 we prove a lemma, which plays an important role in the proofs of the main theorems. In Section 3, we prove the main theorems. We prove Lemma 1.1 in Appendix A

\section{Preliminaries}

Fix $l \in \mathbb{N}$ satisfying $l \geq 2 q+1$, where $q:=p /(p-1)$. Let $\eta \in C_{0}^{1}([0, \infty))$ and $\phi \in C_{0}^{2}\left(\mathbb{R}^{d}\right)$ satisfy

$$
\eta(t):=\left\{\begin{array}{ll}
(1-t)^{2}, & 0 \leq t \leq 1, \\
0, & 1<t,
\end{array} \quad \phi(x):= \begin{cases}1, & |x| \leq 1 / 2, \\
0, & 1 \leq|x|\end{cases}\right.
$$

and $0 \leq \phi \leq 1$. We note that $\eta$ satisfies

$$
\int_{t}^{\infty} \eta(s)^{l} d s \leq C \eta(t)^{l}
$$

for any $t \in[0, \infty)$ where $C$ is a positive constant. Indeed, for $t \geq 0$,

$$
\int_{t}^{\infty} \eta(s)^{l} d s=\int_{t \wedge 1}^{1}(1-s)^{2 l} d s=\frac{1}{2 l+1}\{1-(t \wedge 1)\}^{2 l+1} \leq \frac{1}{2 l+1} \eta(t)^{l} .
$$

We define the test function $\psi_{\tau} \in C_{0}^{2}\left([0, T) \times \mathbb{R}^{d}\right)$ for $\tau \in(0, T)$ by

$$
\psi_{\tau}(t, x):=-\left(\int_{t}^{\infty} \eta\left(\frac{s}{\tau}\right)^{l} d s\right) \phi\left(\frac{x}{\tau}\right)^{l} .
$$

The following lemma plays an important role in the proofs of the main theorems.

Lemma 2.1. Let $s \geq 0, u_{0} \in H^{s}\left(\mathbb{R}^{d}\right), T>0$, and $l \in \mathbb{N}$ with $l \geq 2 q+1$ where $q:=p /(p-1)$. We assume $\operatorname{Re}\left(\bar{\lambda} u_{0}\right)=0$. Let $u$ be a weak solution to (1.1) on $[0, T)$. Then, there exists a positive constant $C=C(\lambda, p, d, l)>0$ such that

$$
-\int_{\mathbb{R}^{d}} v(0, x) \phi\left(\frac{x}{\tau}\right)^{l} d x \leq C \tau^{d+1-q}+m^{2 q} C \tau^{d+1+q},
$$

for any $\tau \in(0, T)$ where $v=\operatorname{Im}\left(\bar{\lambda} u_{0}\right)$. 
Proof. Let $T>0$ and let $u$ be a weak solution to (1.1) on [0,T). By Lemma 1.1, $u$ is also a weak solution to (1.7) on $[0, T)$. We set

$$
I(\tau):=\int_{0}^{T}\left(|u(t)|^{p} \mid \partial_{t} \psi_{\tau}(t)\right) d t
$$

By substituting $\psi_{\tau}$ for $\varphi$ in Definition 1.2 and using the assumption $\operatorname{Re}\left(\bar{\lambda} u_{0}\right)=0$, we obtain

$$
\begin{aligned}
-\left(v(0) \mid \partial_{t} \psi_{\tau}(0)\right) & =-|\lambda|^{2} I(\tau)+\int_{0}^{T}\left(v(t) \mid \partial_{t}^{2} \psi_{\tau}-\Delta \psi_{\tau}+m^{2} \psi_{\tau}\right) d t \\
& =-|\lambda|^{2} I(\tau)+K_{1}+K_{2}+m^{2} K_{3},
\end{aligned}
$$

where

$$
K_{1}:=\int_{0}^{T}\left(v(t) \mid \partial_{t}^{2} \psi_{\tau}\right) d t, \quad K_{2}:=-\int_{0}^{T}\left(v(t) \mid \Delta \psi_{\tau}\right) d t, \quad K_{3}:=\int_{0}^{T}\left(v(t) \mid \psi_{\tau}\right) d t
$$

We estimate $K_{1}, K_{2}$, and $K_{3}$. First, we consider $K_{1}$. By $l-1>l / p$ and the Hölder inequality,

$$
\begin{aligned}
\left|K_{1}\right| & =l \tau^{-1}\left|\int_{0}^{\tau} \int_{|x| \leq \tau} v(t) \eta^{\prime}\left(\frac{t}{\tau}\right) \eta\left(\frac{t}{\tau}\right)^{l-1} \phi\left(\frac{x}{\tau}\right)^{l} d x d t\right| \\
& \leq C \tau^{-1} \int_{0}^{\tau} \int_{|x| \leq \tau}|v(t)| \eta\left(\frac{t}{\tau}\right)^{l-1} \phi\left(\frac{x}{\tau}\right)^{l} d x d t \\
& \leq C \tau^{-1} \int_{0}^{\tau} \int_{|x| \leq \tau}|v(t)| \eta\left(\frac{t}{\tau}\right)^{l / p} \phi\left(\frac{x}{\tau}\right)^{l / p} d x d t \\
& \leq C \tau^{-1}\left(\int_{0}^{\tau} \int_{|x| \leq \tau}|v(t)|^{p} \eta\left(\frac{t}{\tau}\right)^{l} \phi\left(\frac{x}{\tau}\right)^{l} d x d t\right)^{1 / p}\left(\int_{0}^{\tau} \int_{|x| \leq \tau} d x d t\right)^{1 / q} \\
& =C \tau^{(d+1) / q-1}\left(\int_{0}^{\tau} \int_{|x| \leq \tau}|v(t)|^{p} \partial_{t} \psi_{\tau} d x d t\right)^{1 / p} \\
& \leq C \tau^{(d+1) / q-1} I(\tau)^{1 / p} .
\end{aligned}
$$

Second, we consider $K_{2}$. Note that the following holds by (2.2):

$$
\int_{t}^{\infty} \eta\left(\frac{s}{\tau}\right)^{l} d s=\tau \int_{t / \tau}^{\infty} \eta(s)^{l} d s \leq C \tau \eta\left(\frac{t}{\tau}\right)^{l}
$$


By $l-2>l / p,(2.4)$, and the Hölder inequality,

$$
\begin{aligned}
\left|K_{2}\right| \leq & C \tau^{-2} \int_{0}^{\tau} \int_{|x| \leq \tau}|v(t)|\left|\int_{t}^{\infty} \eta\left(\frac{s}{\tau}\right)^{l} d s\right| \\
& \times\left\{\phi\left(\frac{x}{\tau}\right)^{l-2}\left|(\nabla \phi)\left(\frac{x}{\tau}\right)\right|^{2}+\phi\left(\frac{x}{\tau}\right)^{l-1}\left|(\Delta \phi)\left(\frac{x}{\tau}\right)\right|\right\} d x d t \\
\leq & C \tau^{-2} \int_{0}^{\tau} \int_{|x| \leq \tau}|v(t)|\left(\int_{t}^{\infty} \eta\left(\frac{s}{\tau}\right)^{l} d s\right) \phi\left(\frac{x}{\tau}\right)^{l-2} d x d t \\
\leq & C \tau^{-1} \int_{0}^{\tau} \int_{|x| \leq \tau}|v(t)| \eta\left(\frac{t}{\tau}\right)^{l} \phi\left(\frac{x}{\tau}\right)^{l-2} d x d t \\
\leq & C \tau^{-1} \int_{0}^{\tau} \int_{|x| \leq \tau}|v(t)| \eta\left(\frac{t}{\tau}\right)^{l / p} \phi\left(\frac{x}{\tau}\right)^{l / p} d x d t \\
\leq & C \tau^{(d+1) / q-1} I(\tau)^{1 / p} .
\end{aligned}
$$

Finally, we consider $K_{3}$. By $l>l / p$, (2.4), and the Hölder inequality,

$$
\begin{aligned}
\left|K_{3}\right| & \leq \int_{0}^{\tau} \int_{|x| \leq \tau}|v(t)|\left|\int_{t}^{\infty} \eta\left(\frac{s}{\tau}\right)^{l} d s\right| \phi\left(\frac{x}{\tau}\right)^{l} d x d t \\
& \leq C \tau \int_{0}^{\tau} \int_{|x| \leq \tau}|v(t)| \eta\left(\frac{t}{\tau}\right)^{l} \phi\left(\frac{x}{\tau}\right)^{l} d x d t \\
& \leq C \tau^{(d+1) / q+1} I(\tau)^{1 / p} .
\end{aligned}
$$

By these estimates and the Young inequality, we obtain

$$
\begin{aligned}
-\left(v(0) \mid \partial_{t} \psi_{\tau}(0)\right) & \leq-|\lambda|^{2} I(\tau)+C \tau^{(d+1) / q-1} I(\tau)^{1 / p}+m^{2} C \tau^{(d+1) / q+1} I(\tau)^{1 / p} \\
& \leq C \tau^{d+1-q}+m^{2 q} C \tau^{d+1+q} .
\end{aligned}
$$

Therefore, we obtain the statement.

\section{Proofs of the MAIN Theorems}

In this section, we prove the main theorems. First, we prove Theorem 1.2

Proof of Theorem 1.2. By the assumption for the initial data,

$$
\begin{aligned}
-\left(v(0) \mid \partial_{t} \psi_{\tau}(0)\right) & =-\int_{\mathbb{R}^{d}} v(0, x) \phi\left(\frac{x}{\tau}\right)^{l} d x \\
& \geq \mu \int_{|x| \leq 1}|x|^{-k} \phi\left(\frac{x}{\tau}\right)^{l} d x \\
& \geq \mu \tau^{d-k} \int_{|y| \leq \frac{1}{\tau}}|y|^{-k} \phi(y)^{l} d y .
\end{aligned}
$$

Let $L(\tau):=\int_{|y| \leq \frac{1}{\tau}}|y|^{-k} \phi(y)^{l} d y$. Then, by Lemma 2.1, we get

$$
\mu \leq(L(\tau))^{-1}\left(C \tau^{k+1-q}+m^{2 q} C \tau^{k+1+q}\right) .
$$

Claim. There exists $\mu_{0}$ such that $T \leq 2$ if $\mu>\mu_{0}$.

We suppose $T>2$ and $\mu>\mu_{0}$. Taking $\tau=2, \mu$ satisfies

$$
\mu \leq(L(2))^{-1}\left(C 2^{k+1-q}+m^{2 q} C 2^{k+1+q}\right)=: \mu_{0} .
$$


Noting that $L(2)$ is a finite positive constant, this contradicts $\mu>\mu_{0}$. Hence, the Claim is true.

Let $\mu>\mu_{0} . L(\tau)>L(2)$ for any $\tau<T(<2)$ since $L$ is decreasing. For any $\tau<T$,

$$
\begin{aligned}
\mu & \leq(L(\tau))^{-1}\left(C \tau^{k+1-q}+m^{2 q} C \tau^{k+1+q}\right) \\
& \leq(L(2))^{-1}\left(C \tau^{k+1-q}+m^{2 q} C 2^{2 q} \tau^{k+1-q}\right) \\
& \leq C \tau^{k+1-q} .
\end{aligned}
$$

Hence, $\tau \leq C \mu^{-1 /(q-k-1)}$ for any $\tau<T$, where $C$ depends on $k, m, p$, and $\lambda$. We note $q-k-1>0$ since $k<d / 2-s \leq 1 /(p-1)$. Taking the limit $\tau \rightarrow T$, we obtain

$$
T \leq C \mu^{-1 /(q-k-1)} \text {. }
$$

This completes the proof.

Next, we prove Theorem 1.3

Proof of Theorem 1.3. By the same argument as in the proof of Theorem 1.2, for $\tau \in(0, T \wedge 2)$,

$$
\mu \leq C \tau^{k+1-q},
$$

where $C$ depends on $k, m, p$, and $\lambda$. We note $k+1-q>0$ since $1 /(p-1)<k<$ $d / 2-s$. Taking the limit $\tau \rightarrow 0$, we obtain $\mu=0$. This completes the proof.

Finally, we prove Theorem 1.4

Proof of Theorem 1.4. Let $\varepsilon_{0}$ satisfy $C_{0} \varepsilon_{0}^{-1 /(q-k-1)}=2$ where $C_{0}$ is defined later. First, we consider the case of $\mu>\varepsilon_{0}$. We assume that $T>2$. By the assumption for the initial data, for any $\tau \in(0, T)$,

$$
\begin{aligned}
-\left(v(0) \mid \partial_{t} \psi_{\tau}(0)\right) & =-\int_{\mathbb{R}^{d}} v(0, x) \phi\left(\frac{x}{\tau}\right)^{l} d x \\
& \geq \mu \int_{|x| \geq 1}|x|^{-k} \phi\left(\frac{x}{\tau}\right)^{l} d x \\
& \geq \mu \tau^{d-k} \int_{|y| \geq \frac{1}{\tau}}|y|^{-k} \phi(y)^{l} d y .
\end{aligned}
$$

Let $M(\tau):=\int_{|y| \geq \frac{1}{\tau}}|y|^{-k} \phi(y)^{l} d y$. For any $\tau \in(2, T), M(\tau) \geq M(2)>0$ since $M$ is increasing. Therefore, by Lemma 2.1 and $m=0$, we get

$$
M(2) C \mu \leq \tau^{k+1-q},
$$

for $\tau \in(2, T)$. We set $C_{0}:=(M(2) C)^{-1 /(q-k-1)}$. We note $q-k-1>0$ since $d / 2<k \leq 1 /(p-1)$. Hence, $\tau \leq C_{0} \mu^{-1 /(q-k-1)}$. Taking the limit $\tau \rightarrow T$, we obtain

$$
T \leq C_{0} \mu^{-1 /(q-k-1)} \leq 2 .
$$

This contradicts $T>2$. Therefore, $T \leq 2$ for $\mu \in\left[\varepsilon_{0}, \infty\right)$. Next, we consider the case of $\mu<\varepsilon_{0}$. If $T \leq 2$, then the statement is true since $2<C_{0} \mu^{-1 /(q-k-1)}$. Hence, it is sufficient to consider the case $T>2$. By the above argument, we obtain $T \leq C_{0} \mu^{-1 /(q-k-1)}$ for $\mu \in\left(0, \varepsilon_{0}\right)$. 


\section{Appendix A. Proof of Lemma 1.1}

Proof. Let $\varphi \in C_{0}^{2}\left([0, T) \times \mathbb{R}^{d}\right)$. Then $\left(m^{2}-\Delta\right)^{1 / 2} \varphi$ and $\partial_{t} \varphi$ belong to $X_{T}$. By taking the imaginary part of (1.6) with $\varphi$ replaced by $\lambda\left(m^{2}-\Delta\right)^{1 / 2} \varphi$,

$$
\begin{aligned}
\operatorname{Im} \int_{0}^{T}\left(\bar{\lambda} u(t) \mid i \partial_{t}\left(m^{2}-\Delta\right)^{1 / 2} \varphi \pm\right. & \left.\left(m^{2}-\Delta\right) \varphi\right) d t \\
& =\left(\operatorname{Re}\left(\bar{\lambda} u_{0}\right) \mid\left(m^{2}-\Delta\right)^{1 / 2} \varphi(0)\right), \\
\int_{0}^{T}\left(\operatorname{Re}(\bar{\lambda} u) \mid \partial_{t}\left(m^{2}-\Delta\right)^{1 / 2} \varphi\right) d t & \mp \int_{0}^{T}\left(v(t) \mid\left(m^{2}-\Delta\right) \varphi\right) d t \\
& =-\left(\operatorname{Re}\left(\bar{\lambda} u_{0}\right) \mid\left(m^{2}-\Delta\right)^{1 / 2} \varphi(0)\right) .
\end{aligned}
$$

On the other hand, by taking the real part of (1.6) with $\varphi$ replaced by $\lambda \partial_{t} \varphi$,

$$
\begin{aligned}
& \operatorname{Re} \int_{0}^{T}\left(\bar{\lambda} u(t) \mid i \partial_{t}^{2} \varphi \pm\left(m^{2}-\Delta\right)^{1 / 2} \partial_{t} \varphi\right) d t \\
&=-\left(v(0) \mid \partial_{t} \varphi(0)\right)+|\lambda|^{2} \int_{0}^{T}\left(|u(t)|^{p} \mid \partial_{t} \varphi(t)\right) d t \\
& \int_{0}^{T}\left(v \mid \partial_{t}^{2} \varphi\right) d t \pm \int_{0}^{T}\left(\operatorname{Re}(\bar{\lambda} u) \mid\left(m^{2}-\Delta\right)^{1 / 2} \partial_{t} \varphi\right) d t \\
&=-\left(v(0) \mid \partial_{t} \varphi(0)\right)+|\lambda|^{2} \int_{0}^{T}\left(|u(t)|^{p} \mid \partial_{t} \varphi(t)\right) d t .
\end{aligned}
$$

By combining these equalities, we get

$$
\begin{array}{r}
\int_{0}^{T}\left(v \mid \partial_{t}^{2} \varphi\right) d t+\int_{0}^{T}\left(v(t) \mid\left(m^{2}-\Delta\right) \varphi\right) d t \mp\left(\operatorname{Re}\left(\bar{\lambda} u_{0}\right) \mid\left(m^{2}-\Delta\right)^{1 / 2} \varphi(0)\right) \\
=-\left(v(0) \mid \partial_{t} \varphi(0)\right)+|\lambda|^{2} \int_{0}^{T}\left(|u(t)|^{p} \mid \partial_{t} \varphi(t)\right) d t .
\end{array}
$$

Therefore, this completes the proof.

\section{REFERENCES}

[1] Juan P. Borgna and Diego F. Rial, Existence of ground states for a one-dimensional relativistic Schrödinger equation, J. Math. Phys. 53 (2012), no. 6, 062301, 19, DOI 10.1063/1.4726198. MR.2977673

[2] Thierry Cazenave, Semilinear Schrödinger equations, Courant Lecture Notes in Mathematics, vol. 10, New York University, Courant Institute of Mathematical Sciences, New York; American Mathematical Society, Providence, RI, 2003. MR2002047 (2004j:35266)

[3] K. Fujiwara and T. Ozawa, Remarks on global solutions to the Cauchy problem for semirelativistic equations with power type nonlinearrity, preprint.

[4] Masahiro Ikeda and Takahisa Inui, Small data blow-up of $L^{2}$ or $H^{1}$-solution for the semilinear Schrödinger equation without gauge invariance, J. Evol. Equ. 15 (2015), no. 3, 571-581, DOI 10.1007/s00028-015-0273-7. MR.3394699

[5] Masahiro Ikeda and Takahisa Inui, Some non-existence results for the semilinear Schrödinger equation without gauge invariance, J. Math. Anal. Appl. 425 (2015), no. 2, 758-773, DOI 10.1016/j.jmaa.2015.01.003. MR.3303890

[6] Masahiro Ikeda and Yuta Wakasugi, A note on the lifespan of solutions to the semilinear damped wave equation, Proc. Amer. Math. Soc. 143 (2015), no. 1, 163-171, DOI 10.1090/S0002-9939-2014-12201-5. MR.3272741 
[7] Masahiro Ikeda and Yuta Wakasugi, Small-data blow-up of $L^{2}$-solution for the nonlinear Schrödinger equation without gauge invariance, Differential Integral Equations 26 (2013), no. 11-12, 1275-1285. MR3129009

[8] Joachim Krieger, Enno Lenzmann, and Pierre Raphaël, Nondispersive solutions to the $L^{2}$ critical half-wave equation, Arch. Ration. Mech. Anal. 209 (2013), no. 1, 61-129, DOI 10.1007/s00205-013-0620-1. MR 3054599

[9] Hiroyuki Takamura and Kyouhei Wakasa, The sharp upper bound of the lifespan of solutions to critical semilinear wave equations in high dimensions, J. Differential Equations 251 (2011), no. 4-5, 1157-1171, DOI 10.1016/j.jde.2011.03.024. MR2812585(2012f:35364)

[10] Grozdena Todorova and Borislav Yordanov, Critical exponent for a nonlinear wave equation with damping, J. Differential Equations 174 (2001), no. 2, 464-489, DOI 10.1006/jdeq.2000.3933. MR.1846744(2002k:35218)

[11] Borislav T. Yordanov and Qi S. Zhang, Finite time blow up for critical wave equations in high dimensions, J. Funct. Anal. 231 (2006), no. 2, 361-374, DOI 10.1016/j.jfa.2005.03.012. MR2195336 (2006j:35169)

[12] Qi S. Zhang, A blow-up result for a nonlinear wave equation with damping: the critical case (English, with English and French summaries), C. R. Acad. Sci. Paris Sér. I Math. 333 (2001), no. 2, 109-114, DOI 10.1016/S0764-4442(01)01999-1. MR.1847355 (2003d:35189)

[13] Yi Zhou, Blow up of solutions to semilinear wave equations with critical exponent in high dimensions, Chin. Ann. Math. Ser. B 28 (2007), no. 2, 205-212, DOI 10.1007/s11401-0050205-x. MR2316656(2008a:35201)

Department of Mathematics, Kyoto University, Kyoto 60-5802, Japan

E-mail address: inui@math.kyoto-u.ac.jp 